\title{
Anticipatory Control of Grasping: Independence of Sensorimotor Memories for Kinematics and Kinetics
}

\author{
Jamie R. Lukos, ${ }^{1}$ Caterina Ansuini, ${ }^{3}$ and Marco Santello ${ }^{1,2}$ \\ ${ }^{1}$ Department of Kinesiology and ${ }^{2}$ The Harrington Department of Bioengineering, Arizona State University, Tempe, Arizona 85287, and ${ }^{3}$ Dipartimento di \\ Psicologia Generale, Università di Padova, 35131, Padova, Italy
}

We have recently provided evidence for anticipatory grasp control mechanisms in the kinematic domain by showing that subjects modulate digit placement on an object based on its center of mass (CM) when it can be anticipated (Lukos et al., 2007). This behavior relied on sensorimotor memories about digit contact points and forces required for optimal manipulation. We found that accurate sensorimotor memories depended on the acquisition of implicit knowledge about object properties associated with repeated manipulations of the same object.

Whereas implicit knowledge of object properties is essential for anticipatory grasp control, the extent to which subjects can use explicit knowledge to accurately scale digit forces in an anticipatory manner is controversial. Additionally, it is not known whether subjects are able to use explicit knowledge of object properties for anticipatory control of contact points. We addressed this question by asking subjects to grasp and lift an object while providing explicit knowledge of object CM location as visual or verbal cues. Contact point modulation and object roll, a measure of anticipatory force control, were assessed using blocked and random CM presentations. We found that explicit knowledge of object CM enabled subjects to modulate contact points. In contrast, subjects could not minimize object roll in the random condition to the same extent as in the blocked when provided with a verbal or visual cue. These findings point to a dissociation in the effect of explicit knowledge of object properties on grasp kinematics versus kinetics, thus suggesting independent anticipatory processes for grasping.

Key words: reaching; explicit knowledge; implicit knowledge; fingertip; contact points; motor learning

\section{Introduction}

Studies of grasping have reported that one or two object lifts are often sufficient for the formation of sensorimotor memories allowing for anticipatory digit force modulation to object properties such as weight, texture, or center of mass (CM) (Gordon et al., 1993; Burstedt et al., 1999; Johansson et al., 1999; Reilmann et al., 2001; Rearick and Santello, 2002). We have reported evidence for the presence of anticipatory control mechanisms also for contact points on an object. Specifically, the spatial distribution of contact points was modulated when subjects had implicit knowledge of object CM location resulting from direct somatosensory information acquired through lifting the object. This knowledge allowed subjects to anticipate the necessary forces required to minimize object roll during lift. However, when CM could not be anticipated,

\footnotetext{
Received Aug. 8, 2008; revised 0ct. 8, 2008; accepted 0ct. 9, 2008.

This work was partially supported by National Institutes of Health Bioengineering Research Partnership Grant R01-NS050265 and National Science Foundation Grant BCS-0819547. We thank Dr. Thanassis Rikakis, Todd Ingalls, Jodi James, and Siew Wong from Arts, Media, and Engineering for use of their facilities, technical support, data collection, and processing. We thank Dr. Steve Helms Tillery, Dr. Miya Rand, Dr. Peter Killeen, and the Neural Control of Movement Laboratory members for their comments on a previous version of this manuscript and Brian Pummell and Lisa Platero for help with data processing. We also thank the two anonymous reviewers for their insightful comments.

Correspondence should be addressed to Marco Santello, Department of Kinesiology, Arizona State University, Tempe, AZ 85287-0404. E-mail: marco.santello@asu.edu.

D0I:10.1523/JNEUROSCI.4335-08.2008

Copyright $\odot 2008$ Society for Neuroscience $\quad$ 0270-6474/08/2812765-10\$15.00/0
}

subjects used a "default" distribution of contact points and object roll was significantly larger (Lukos et al., 2007).

Whereas the above research focused on the effect of implicit knowledge of object properties on grasp performance, much less is known about subjects' ability to use explicit knowledge for the planning and execution of grasp. For example, subjects are unable to use arbitrary visual cues to accurately scale digit forces to object CM (Salimi et al., 2003). Similarly, verbal cues about the magnitude of impending collisions between a pendulum and a grasped object (Turrell et al., 1999) or object weight (Flanagan and Beltzner, 2000) did not allow subjects to properly scale digit forces. These studies found that anticipatory force control mechanisms are more effective when resulting from motor experience than through explicit knowledge of object properties. In contrast, other studies have reported that arbitrary visual cues are just as effective as motor experience for force scaling to object weight (Cole and Rotella, 2002; Chouinard et al., 2005; Nowak et al., 2007; Ameli et al., 2008). It should be emphasized that all of these studies focused on the effect of explicit knowledge on force planning, ignoring contact point modulation as an integral part of anticipatory control of manipulation (Friedman and Flash, 2007).

The present study was designed to determine the effect of explicit knowledge of object CM on anticipatory control of digit contact points and forces. Specifically, we wanted to 
quantify the extent to which anticipatory control of grasping, based on sensorimotor memories derived from previous manipulations (implicit knowledge), could be replaced by providing subjects with explicit knowledge about CM. This question was addressed by providing verbal or visual cues while randomly changing CM location from trial to trial, hence preventing subjects from developing stable sensorimotor memories. This random condition was compared with a blocked condition in which CM was invariant across trials. Based on the aforementioned evidence of subjects' inability to use cues for anticipatory control of digit forces to object CM, we hypothesized that explicit knowledge of CM would not allow subjects to (1) appropriately anticipate the forces necessary to minimize object roll or (2) modulate contact points to CM location.

\section{Materials and Methods \\ Subjects}

Twelve right-handed healthy subjects (six female and six male, ages $21-43$ years) with normal or corrected-to-normal vision participated in the experiments. All subjects were naive to the experimental purpose of the study. Each subject gave informed consent to participate in the study according to the Declaration of Helsinki, and the experimental procedures were approved by the Institutional Review Board at Arizona State University.

\section{Experimental task}

We asked subjects to reach, grasp, lift, and replace a cylindrical object with their right hand. The cylinder of the object was made of sturdy cardboard covered with black matte tape for uniform texture. Affixed to the cylinder was a wooden rectangular base, making the object look like an inverted "T" (Fig. 1A). The cylinder was aligned with the subject's midline (Fig. $1 D$ ). The $\mathrm{CM}$ of the object was changed by adding a mass $(0.4 \mathrm{~kg})$ in the base of the object in one of three slots, i.e., left, center, or right (Fig. 1A). The total weight of the object with the added mass was 0.81 $\mathrm{kg}$.

Subjects were asked to perform this task by using the fingertips of all digits, but no instructions were given on where to grasp the object along the cylinder. The only task requirement was to minimize object roll while lifting the object vertically $(\sim 15-20 \mathrm{~cm}$ above the table). Subjects performed the task at a self-selected speed. These instructions were the same as those used in our previous study (Lukos et al., 2007), in which subjects were not provided with explicit knowledge about the CM location of the object.

In the present study, we designed two experiments to quantify the effect of explicit knowledge about CM location on planning multidigit contact points and forces. Specifically, we presented subjects with either a verbal or visual cue about object CM ("verbal cue" and "visual cue" experiments; see below). Within each experiment, subjects were informed that object CM was going to be changed either across blocks of trials ("blocked" condition) or in a pseudorandomized order from trial to trial ("random" condition). Note that random trial-to-trial changes prevent planning of digit forces and contact points as a function of object CM location because implicit knowledge gained from trial $n$ cannot be used on trial $n+1$ (Lukos et al., 2007). Therefore, the ultimate goal of both experimental designs was to determine whether subjects could use cues (explicit knowledge) in the random condition to anticipate digit contact points and forces to the
B

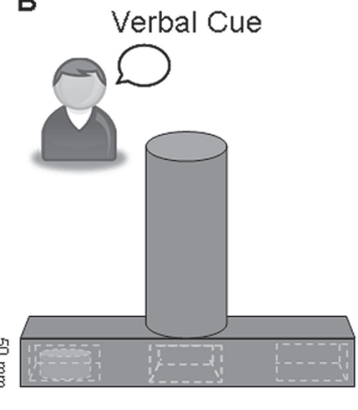

C

Visual Cue
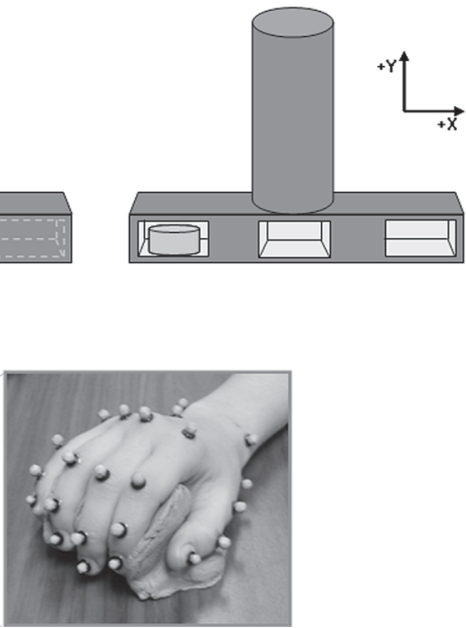

Figure 1. Experimental setup. A shows the object (frontal plane) used for our reach-to-grasp task, the location of the reflective depicted from the subject's perspective. D shows the approximate location of the cameras and the workspace used for motion capture (top view; figure is not to scale). The photo shows start hand posture together with marker placement on the hand and wrist.

same extent as when they gained information about the object through repeated manipulations (implicit knowledge) in the blocked condition. Using two types of cues also afforded us the opportunity to test whether their effect(s) on anticipatory control of contact points and digit forces is specific to the type of cue being used to convey CM location.

Verbal cue experiment. Before reach onset, we told subjects the location of the added mass, e.g., "The mass is now on the left." Before the experiment started, subjects were told that the designation of "left" and "right" was relative to their frame of reference, i.e., thumb and finger side of the object, respectively. For this experimental condition, subjects were not allowed to see the location of the added mass (Fig. $1 B$ ).

Visual cue experiment. Subjects were allowed to view the location of the mass, added at the base of the object, before movement onset as well as for the entire duration of the trial (Fig. 1C). No verbal information about CM location was given to the subjects.

The verbal cue and visual cue experiments were run in the same session as the "no cue" experiment whose results were described previously by Lukos et al. (2007). Note that the predictability conditions in the present study (blocked and random) are equivalent to "predictable" and "unpredictable" conditions, respectively, by Lukos et al. (2007). Each experiment was run as a block, but the order of the experiments and conditions was counterbalanced across subjects (see Fig. 4). Subjects performed 15 experimental trials (3 CM locations $\times$ 5 repetitions) for both the random and blocked condition for a total of 30 trials per experiment. The order of CM blocks and CM locations was also randomized across subjects for the blocked and random conditions, respectively (see Fig. 4, gray and white boxes). Furthermore, the presentation of object CM was designed such that subjects never experienced the same CM location when transitioning from one condition or experiment to another. We used this design to prevent transfer of implicit knowledge of a given object CM to a different condition or experiment (statistical analyses confirmed that no transfer occurred; see Results). Because subjects could not anticipate CM location for the first trial of the blocked condition based on previous 
experience, we gave subjects one practice trial followed by five experimental trials for each CM location.

\section{Data recording}

The experimental procedures used for recording and processing hand and object kinematics have been described in detail by Lukos et al. (2007). Briefly, movement of the hand and object was recorded using a near-infrared camera motion capture system (Eagle-4 Digital System; frame rate, $100 \mathrm{~Hz}$; shutter speed, 800 frames/s; Motion Analysis Corporation). Seven cameras were placed in a semicircle $1-1.5 \mathrm{~m}$ from the subject, who was seated at a table where the object was placed (Fig. $1 D$, left). Each subject was outfitted with lightweight retro-reflective markers (4-6 $\mathrm{mm}$ in diameter) on the hand (Fig. $1 D$, right). The spatial accuracy of the recording system was $\sim 0.15 \mathrm{~mm}$ in the $x, y$, and $z$ planes.

Analysis of hand kinematics focuses on the spatial distribution of digit contact points as defined by the marker placed on the center of each fingernail (see photo in Fig. $1 D$, right). Markers were also placed on the top and sides of the object (Fig. $1 \mathrm{~A}$ ) to determine (1) object lift onset, (2) object roll, and (3) the spatial distribution of the fingertips relative to the object (see below). Because our protocol was designed to let subjects choose digit placement, we could not use force sensors at fixed locations on the object to measure individual digit forces. However, the behavioral outcome, peak object roll, is an indirect measure of anticipatory force control, with smaller rolls being evidence of more accurate digit force scaling to the expected external torque after onset of object lift (Salimi et al., 2000, 2003).

\section{Data processing}

Each trial was visually inspected to verify proper marker identification and the absence of movement artifacts (for details, see Lukos et al., 2007). These data were then run through a low-pass Butterworth filter with a 6 Hz cutoff.

Digit kinematics. For the analysis of fingertip location, we measured the anteroposterior and vertical coordinates ( $z$-axis and $y$-axis, respectively) (Fig. $1 C, D$, see spatial frames of reference) of fingertip position at the time of contact with the object. "Contact time" was defined as the time at which the tangential velocity of the marker on the tip of each digit reached its minimum value between the time of peak wrist velocity (Fig. $1 D$, right, defined by a marker on the radius) and object lift onset (defined below). For each trial, we verified accuracy of this algorithm offline by using measures computed from the object model (cylinder diameter) and the hand model (distance between the thumb and the digits). We found that fingertip tangential velocity accurately defined the time of contact between the fingertip and the object (for details, see Lukos et al., 2007). "Contact points" were defined as the anteroposterior and vertical coordinates of fingertip location at contact time. We transformed the $y$ and $z$-coordinates of each fingertip to an object-centered frame of reference by expressing them relative to the $y$ - and $z$-coordinates of the center of the base of the cylinder $(0,0)$.

Object kinematics. "Object lift onset" was defined as the time at which the tangential velocity of the top center marker of the object crossed a velocity threshold of $5 \mathrm{~mm} / \mathrm{s}$ and remained above it for longer than 200 ms (Lukos et al., 2007, their Fig. 2). Initiation of "corrective responses," indicated by object roll reversal, coincides with peak object roll occurring during object lift (for details, see Lukos et al., 2007). We measured "peak object roll" on the $x-y$ plane, defined as the angle between the gravitational vertical and the line connecting top and bottom markers on the cylinder. In most trials, peak object rolls (ranging from approximately $-22^{\circ}$ to $+24^{\circ}$ ) occurred at reaction time latencies, i.e., $\sim 180-250 \mathrm{~ms}$ after object lift onset. However, in a few cases, peak object roll occurred later than these latencies, particularly for the blocked condition, because it resulted from overcompensation of the corrective response. Because we were interested in object roll minimization resulting from anticipatory control mechanisms, as done in our previous work, the behavioral analysis focused on the initial roll occurring before the end of object lift (see Fig. $5 \mathrm{~A}$, vertical solid lines). Last, we measured "time to peak roll" defined as the latency between object lift onset and the time at which peak roll occurred. This variable was analyzed to quantify how long subjects took to generate adequate forces to counteract object roll. Longer times to peak object roll indicate suboptimal planning of digit force distributions. We wrote custom software (Matlab; MathWorks) to compute all of the above variables.

\section{Statistical analyses}

Statistical analysis of contact point modulation and object roll minimization was performed on the experimental trials for the blocked and random conditions for each cue experiment. Averaged data from verbal cue and visual cue experiments were analyzed with separate ANOVAs. We performed each ANOVA with repeated measures with "predictability condition" (blocked vs random) and "CM location" (left, center, and right) as within-subject factors on the following variables: (1) contact point of each digit, (2) peak object roll, and (3) time to peak object roll. Statistical analyses for peak object roll were performed on the absolute value of the data as explained by Lukos et al. (2007). Comparisons of interest were further analyzed using post hoc $t$ tests with Bonferroni's corrections $(\alpha$ level $=0.05$ ). Additional analyses in Results focus on individual trial responses to describe the time course of the learning processes associated with the presence of a cue.

\section{Results \\ Contact point modulation as a function of object CM}

Consistent with our previous work (Lukos et al., 2007), maximum modulation of contact points across CM locations in the anteroposterior dimension (i.e., rotation of the hand around the circumference of the cylinder) was small (verbal cue, from 2.0 $\mathrm{mm}$ at the ring finger to $6.9 \mathrm{~mm}$ at the index finger; visual cue, from $4.0 \mathrm{~mm}$ at the middle finger to $8.3 \mathrm{~mm}$ at the index finger). In contrast, maximum modulation of contact points in the vertical dimension was from $6.2 \mathrm{~mm}$ at the little finger to $18.1 \mathrm{~mm}$ at the thumb for the verbal cue and from $3.4 \mathrm{~mm}$ at the little finger to $27.0 \mathrm{~mm}$ at the thumb for the visual cue (on average, more than a threefold difference in the vertical vs anteroposterior maximum modulation of contact points). Therefore, all analyses presented below were performed only on the vertical fingertip position.

When CM was changed on a trial-to-trial basis, subjects were able to use either cue to associate the expected torque with a distribution of contact points similar to that used when CM was the same across several consecutive trials. Specifically, when the experimenter told the subjects the location of the CM (verbal cue), subjects responded to random trial-to-trial changes in object CM by modulating contact points. In both blocked and random conditions, subjects lowered the thumb and raised the index finger when comparing left with right CM locations (Fig. 2, second column). The same conditions caused weaker modulation of contact points for middle, ring, and little fingers. The ANOVA revealed a main effect of CM location for all five digits (thumb and index finger, $F_{(2,100)}=41.65$ and 21.68 , respectively, both $p<0.0001$; middle, ring, and little fingers, $F_{(2,100)}=10.26,7.069$, and 6.57, respectively, all $p<0.01)$. However, no main effect of predictability condition or significant interaction between CM location and predictability condition was found for any of the digits.

When subjects were allowed to view the location of the added mass (visual cue), we found similar results as those described for the verbal cue experiment. Specifically, contact points of the thumb and index fingers were modulated to CM location in both blocked and random conditions (Fig. 2, third column). The effect of CM location on contact points of the thumb and index finger were much larger than for the remaining digits.

These qualitative observations were confirmed by ANOVA. We found a main effect of CM location on thumb and index finger contact points $\left(F_{(2,100)}=30.96, p<0.0001\right.$ and $F_{(2,100)}=$ $4.41, p<0.05$, respectively) but not on contact points of the other digits. We also found a main effect of predictability condition on 
contact points of most digits (thumb, index, middle, and ring fingers, $F_{(1,50)}=$ $8.92,9.70,6.97$, and 6.47, respectively, all $p<0.05)$. This effect was caused by the slightly higher $(\sim 7 \mathrm{~mm})$ digit positions for the random compared with the blocked condition. However, post hoc analysis revealed that these differences in predictability condition failed to reach statistical significance for any of these digits. No significant interaction was found between predictability condition and CM location.

The relationship between contact points and object CM found for the blocked condition was not identical to that elicited by random CM presentations, this being particularly clear for the visual cue experiment (Fig. 2, third column). Nevertheless, the fact that modulation of thumb and index finger contact points occurred as a function of object CM in the random condition does not support our hypothesis, because it indicates anticipatory control in response to object CM cued either verbally or visually. The lack of significant interaction between CM location and predictability condition in either cue experiment indicates that a similar modulation of contact points to object CM location occurred regardless of blocked versus randomized CM presentation.

Data from the no cue experiment (Lukos et al., 2007) are shown in the first column of Figure 2 for comparison with the data from verbal cue and visual cue experiments. When subjects were given no cue about the CM location, although it could be predicted based on previous experience (blocked condition), the vertical location of fingertip contact points varied depending on CM location. In contrast, in the random condition, subjects chose a similar distribution of contact points regardless of object $\mathrm{CM}$ location resembling that of the center CM location during the blocked condition.

\section{Covariation between pairs of fingertip contact points}

We used linear regression analysis to quantify the extent to which the contact point of a given digit covaried with that of another digit (for more details on the rationale, see Lukos et al., 2007). Figure 3 depicts the covariation patterns between all digit pairs ( $r$ values) as polar plots for each experiment. For each plot, the closer the dot is to the center of the circle $(r=0)$, the greater the independence between pairs of contact points. The linear regression revealed that, when subjects were given a verbal or visual cue about CM location, similar patterns of covariation between pairs of contact points were found in the blocked and random conditions (Fig. 3, middle and right polar plots). Note, however, that data from the no cue experiment revealed dissimilar covariation patterns between blocked and random conditions, i.e., greater inde-

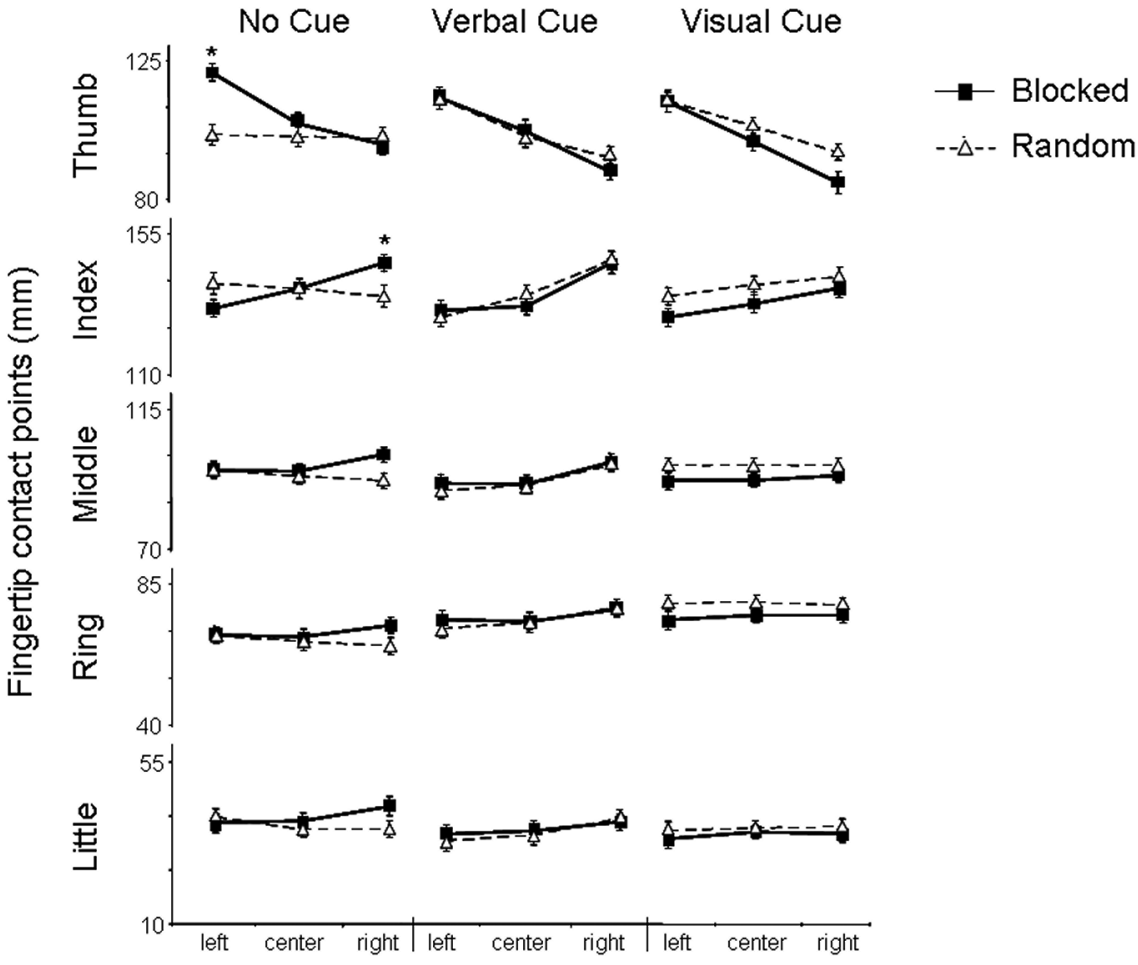

Figure 2. Individual fingertip contact points. Fingertip vertical location relative to the base of the cylinder is shown for each digit as a function of CM location. Data from the no cue (Lukos et al., 2007), verbal cue, and visual cue experiments are shown on the left, middle, and right columns, respectively. Data from blocked (filled squares) and random (open triangles) conditions are shown for each experiment. The range of the vertical axes is the same for all plots to allow comparison across digits. Data are means \pm SEs of all subjects; asterisks represent significant differences $(p<0.05)$ confirmed by post hoc analyses.

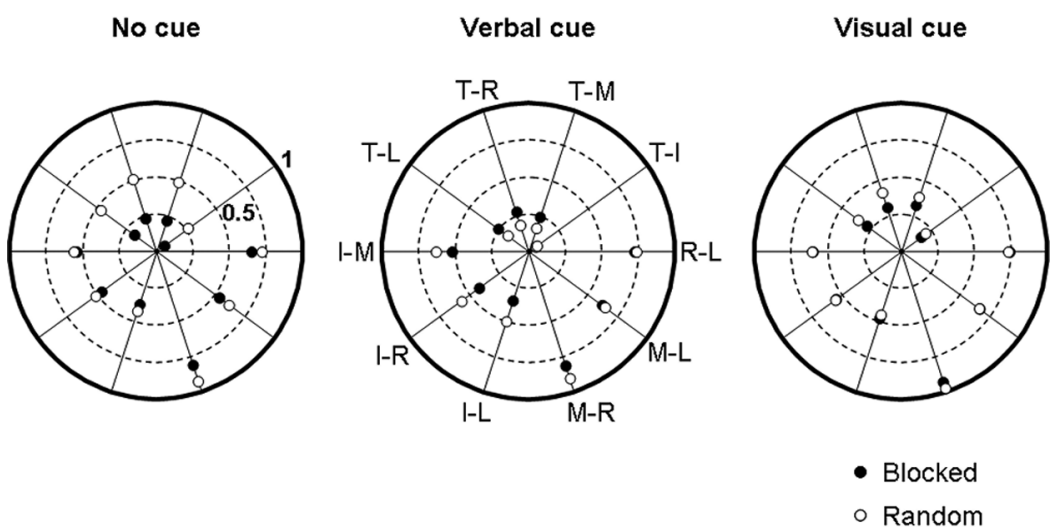

Figure 3. Linear covariation between fingertip contact points. The magnitude of the correlation coefficient (Pearson's $r$ ) computed on the vertical location of the contact points of each digit pair is shown for the no cue (Lukos et al., 2007), verbal cue, and visual cue experiments (left, middle, and right polar plot, respectively). The $r$ values shown in each polar plot were averaged across all subjects. Black and white dots denote blocked and random conditions, respectively. $T, I, M, R$, and $L$ denote thumb, index, middle, ring, and little fingers, respectively. The $r$ values were $z$-normalized before averaging across subjects ( $r$ values closer to 0 indicate greater independence between finger contact point pairs).

pendence in the former than the latter condition (Fig. 3, left polar plot).

\section{Peak object roll minimization}

Figure 4 shows peak object roll plotted throughout the trial sequence from all subjects (labeled as s1 through s12), blocked and random conditions (gray and white boxes, respectively), and no cue, visual cue, and verbal cue experiments (labeled No, Visual, and Verbal, respectively). Several observations can be made about subjects' performance. 

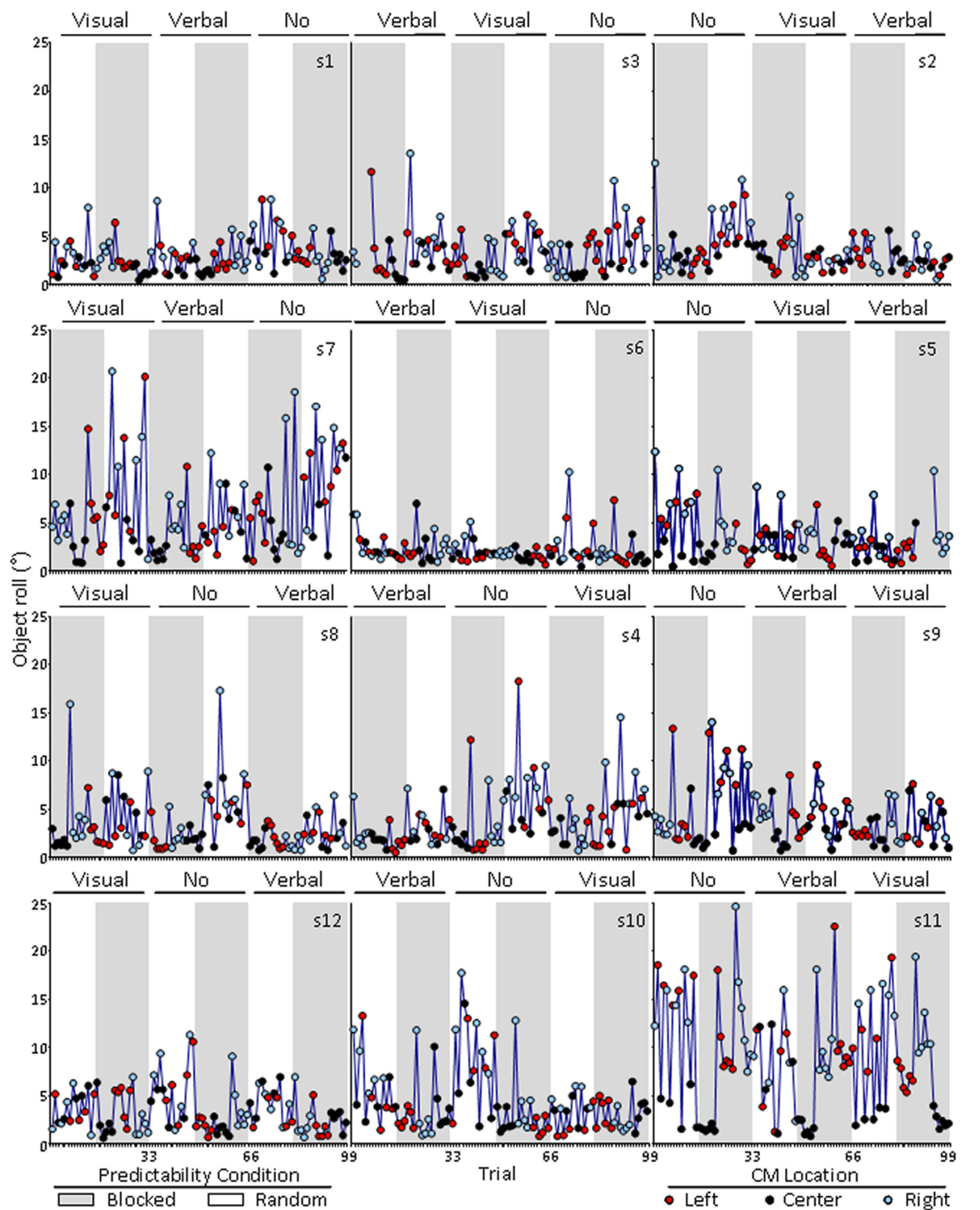

Figure 4. Peak object roll as a function of trial. Each panel shows peak object roll measured for each trial for subjects 1-12 (s1-s12). Data on the left, middle, and right columns denote experimental sessions that started with the visual cue, verbal cue, and no cue experiment, respectively (labeled as Visual, Verbal, and №). For each experiment, trials from blocked and random conditions are indicated by gray and white boxes, respectively. Red, black, and blue symbols denote peak object roll for left, center, and right CM location trials, respectively. Note that the order of experiments, predictability conditions, and CM blocks (blocked condition) was counterbalanced across all subjects.

(1) Within each blocked condition, the first trial (practice trial) of each CM was often associated with the largest peak object roll followed by a sudden drop in magnitude in the succeeding trials. This indicates that subjects were able to use sensorimotor information gained on the practice trial to anticipate object CM location on subsequent trials.

(2) In the no cue experiment, the random sequence of object $\mathrm{CM}$ locations prevents subjects from minimizing object roll to the same extent as the blocked sequence, as indicated by large trial-to-trial fluctuations in peak object roll magnitude. The difference in performance between blocked and random conditions was also found when CM location was cued, although this difference appears to be smaller than in the no cue experiment. This suggests that the cues might have allowed subjects to anticipate object CM location to some extent during the random condition.

(3) Occasionally, peak object rolls during the random sequences were smaller than when transitioning from one $\mathrm{CM}$ to another (on the practice trial) within the blocked sequence, e.g., s3, s8, s9, s11. This might have occurred because, in the blocked trials, subjects were exposed to a sudden change of object CM right after having experienced a different $\mathrm{CM}$ for several consecutive trials.

Statistical analyses revealed that peak object roll was minimized to a greater extent in the blocked condition compared with the random condition in all three experiments (Fig. 5). Providing either a verbal or visual cue about CM location, however, allowed the subject shown in Figure $5 A$ (subject 8 ) to minimize object roll in either of the cued random conditions to an extent that was somewhat intermediate between the performance elicited by the blocked conditions and that associated with the no cue random condition. The statistical analyses below confirmed this pattern to be common to all subjects (Fig. $5 B)$.

When subjects were given either a verbal or visual cue about object CM location, we found a significant main effect of $\mathrm{CM}$ location (verbal cue, $F_{(2,100)}=10.45, p<$ 0.01 ; visual cue, $\left.F_{(2,100)}=15.12, p<0.01\right)$ and predictability condition (verbal cue, $F_{(1,50)}=49.60, p<0.01$; visual cue, $F_{(1,50)}$ $=33.74, p<0.01$ ) (Fig. 5B), as well as a significant interaction between these two factors (verbal cue, $F_{(2,100)}=3.23, p<$ 0.05 ; visual cue, $\left.F_{(2,100)}=4.40, p<0.05\right)$. This interaction was caused by larger peak object rolls in the random conditions when object CM location was on the right (average difference of $2.5^{\circ}$ ). Larger object rolls in the cued random versus blocked conditions were also found for left CM location (average difference of $1.2^{\circ}$ ), but these differences failed to reach statistical significance. A tendency of greater rolls for right versus left CM location in the random condition was also observed in the no cue experiment, although this difference $\left(0.5^{\circ}\right)$ was smaller than for both cue experiments (see above). These findings support our hypothesis that subjects would be unable to use explicit knowledge to anticipate digit forces to the same extent for both random and blocked conditions.

The random condition of the no cue experiment was characterized by significantly larger rolls for left and right CM locations (mean peak roll, $7.8^{\circ}$ and $8.3^{\circ}$, respectively) than for the blocked condition (mean peak roll, $2.7^{\circ}$ and $3.4^{\circ}$, respectively), whereas similar rolls between the two predictability conditions were found for the center CM location $\left(1.7^{\circ}\right.$ and $3.2^{\circ}$, respectively).

\section{Peak object roll distribution}

Figure 6 shows the distribution of object rolls across all trials and pooled across all subjects from each predictability condition and cue experiment. Comparison of the random data between the verbal cue or visual cue data with the no cue data suggests that subjects were better able to minimize object roll with than with- 
out cues about CM location, i.e., fewer trials characterized by large object rolls in either direction. Nevertheless, for either cue experiment, the central region of the peak object roll distribution is higher in the blocked than in the random condition. Therefore, it appears that subjects were not able to use the cues to optimally minimize object roll for the random condition as well as they did for blocked trials. To quantify these observations, two variables were computed from the peak roll distribution of each subject: (1) variance of peak rolls averaged across all CM locations and (2) number of trials in which the object rolled within $\pm 2^{\circ}$ from the vertical.

\section{Variance of peak object roll}

Figure $7 A$ shows data from each subject as well as the mean variance computed across all subjects. Both verbal and visual cues about CM location allowed subjects to better counteract object torque than in the no cue experiment, as revealed by a reduction in the magnitude of peak object roll in the random condition despite trial-to-trial changes in object CM location. $t$ tests revealed similar variances in the peak object roll distributions in the random and blocked conditions when subjects were given either a verbal or visual cue ( $p$ values $>0.05)$, but significant differences were found between the blocked and random conditions of the no cue experiment $\left.t_{(22)}=-3.40, p<0.01\right)$.

\section{Optimal minimization of object roll}

More than half of the trials from the blocked conditions were characterized by very small peak object rolls, i.e., within $\pm 2^{\circ}$ relative to the vertical (Fig. 6 , left column). Therefore, we used it as a measure of optimal minimization of object roll (Fig. 7B). The random condition was characterized by a significantly smaller number of trials in which subjects could minimize object rolls within $\pm 2^{\circ}$ from the vertical compared with the blocked condition, regardless of cues ( $t$ tests, $t_{(22)}=4.28,3.42$, and 2.99 for no cue, verbal cue, and visual cue, respectively; all $p<0.01$ ).

\section{Time to peak object roll}

When subjects were given either a verbal or visual cue, time to peak object roll was similar for both the blocked and random condition (verbal cue, 197 and 205 ms; visual cue, 219 and $211 \mathrm{~ms}$, blocked and random, respectively). Therefore, subjects generated a counteracting torque at similar latencies regardless of predictability condition when cues about CM location were given. In contrast, for the no cue experiment, subjects responded at longer latencies when CM location was randomly changed from trial to trial (182 and 258
A
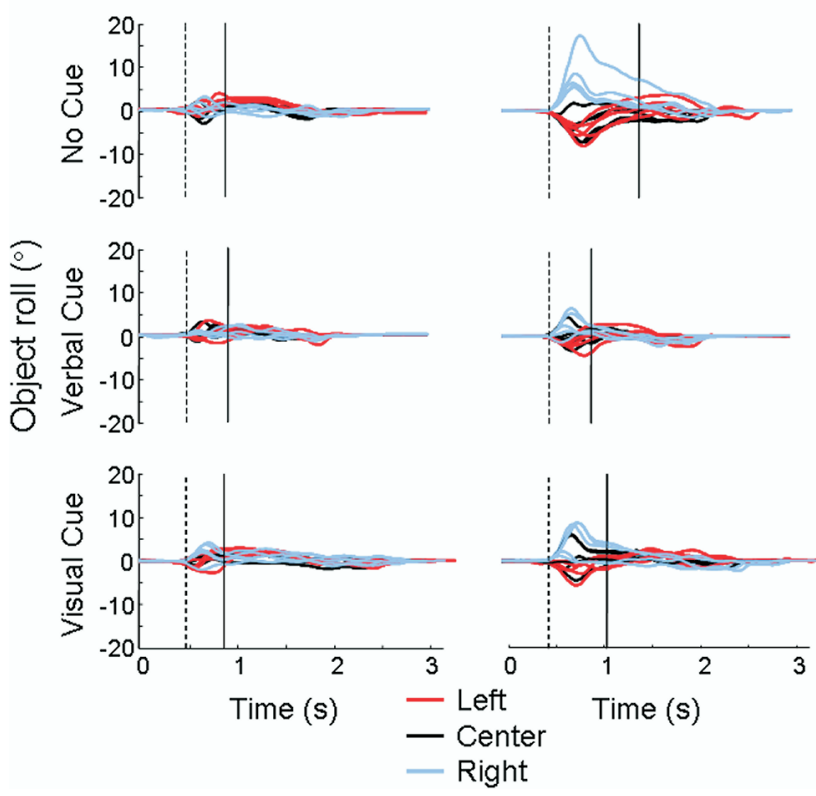

B
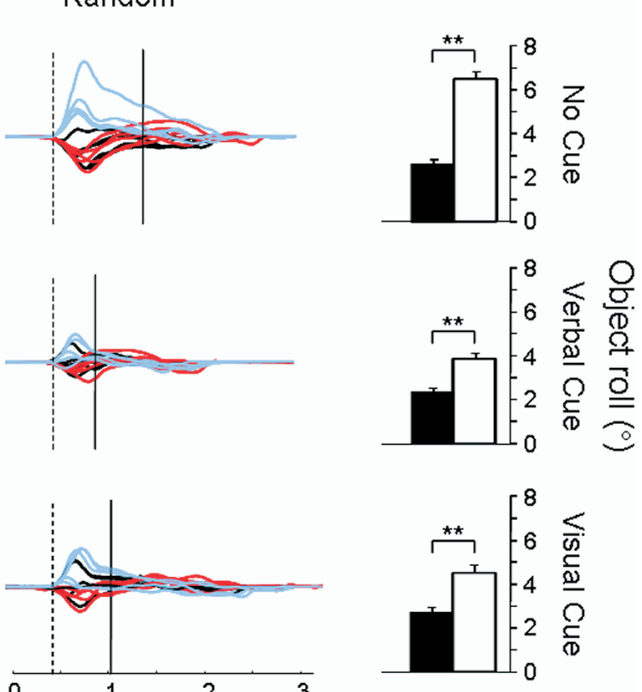

Blocked
Figure 5. Object roll minimization. $\boldsymbol{A}$ shows the time course of object roll for one representative subject (\#8) for each experiment [no cue (Lukos et al., 2007), verbal cue, and visual cue; top, middle, and bottom rows, respectively]. Data from blocked and random CM presentation are shown on left and right columns, respectively. Dashed and solid vertical lines denote object lift onset and average end of lift, respectively (for details, see Lukos et al., 2007). Data are aligned with respect to object lift onset. Positive and negative values denote object rolls toward the subject's fingers and thumb, respectively (see Fig. $1 \mathrm{~A}$ ). $\boldsymbol{B}$ shows peak object roll averaged across all subjects ( \pm SE) for each predictability condition and experiment. ${ }^{* *} p<0.001$, significant main effect of predictability condition.

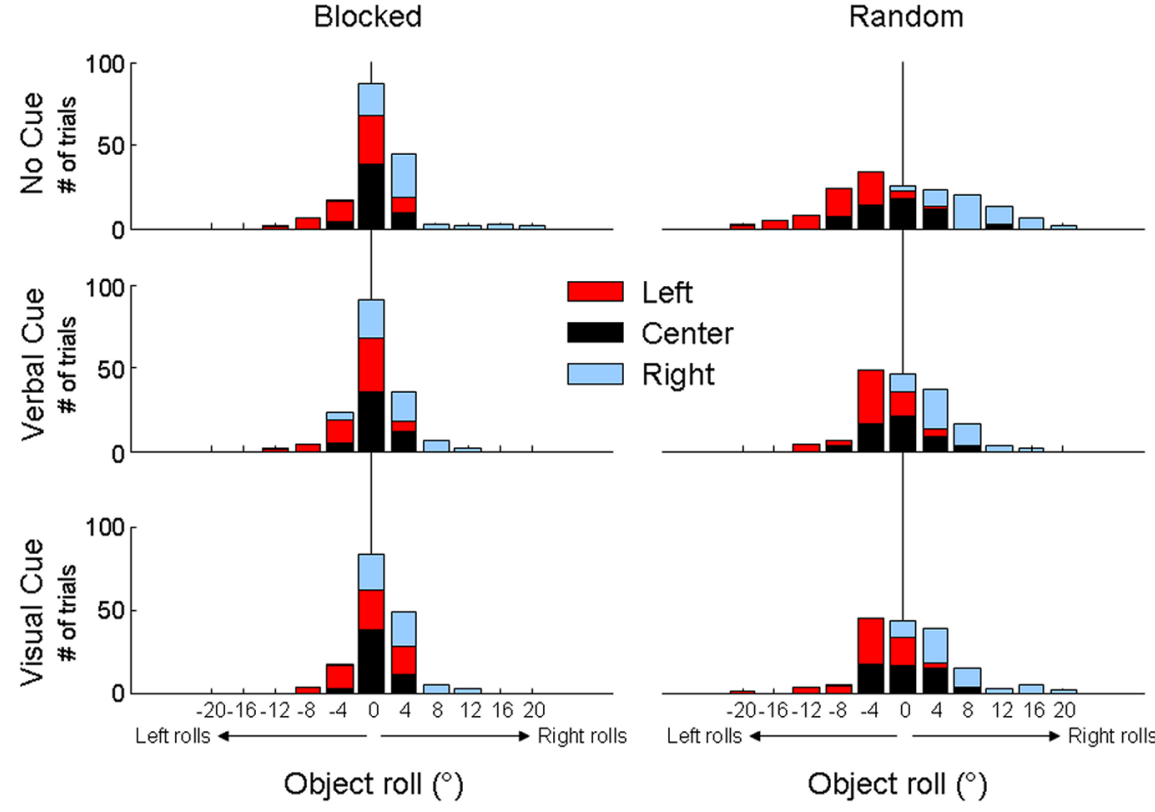

Figure 6. Distributions of peak object roll as a function of object CM location. The trial distributions of peak object roll for no cue (Lukos et al., 2007), verbal cue, and visual cue experiments (top, middle, and bottom rows, respectively) were pooled across all subjects and binned at $4^{\circ}$ intervals. Note, however, that statistical analysis was performed on the peak roll distributions from each subject. The vertical line $\left(0^{\circ}\right)$ denotes gravitational vertical (the bin labeled $0^{\circ}$ represents the number of trials characterized by peak object rolls of $\pm 2^{\circ}$ ). Negative and positive object rolls indicate rolls to the left (thumb side) and right (finger side), respectively (Fig. $1 \mathrm{~A}$ ).

ms, blocked and random, respectively). These effects can be seen in the time course of peak object roll from one representative subject shown in Figure $5 A$.

For the verbal cue experiment, the ANOVA showed no main effect of either CM location or predictability condition on time to 


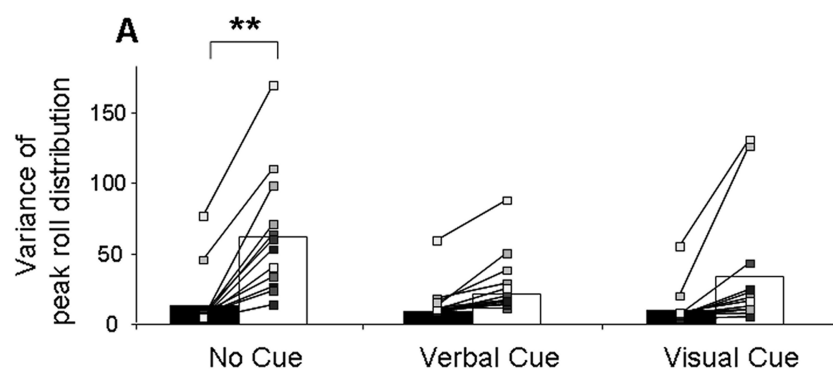

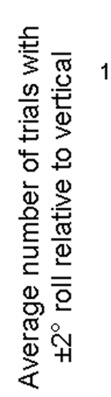

B

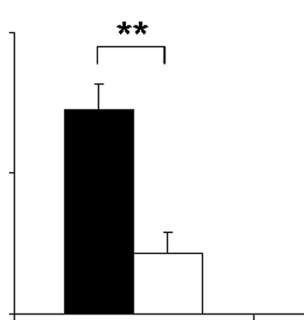

0
5
0
No Cue
Verbal Cue

Visual Cue

Figure 7. Variance of peak object roll and minimization within $\pm 2^{\circ}$ object rolls. $\boldsymbol{A}$ shows the variance of the peak object rol distributions from individual subjects (each symbol denotes data from one subject) and the mean variance averaged across all subjects (bars) for the no cue (Lukos et al., 2007), verbal cue, and visual cue (first, second, and third columns, respectively) for each predictability condition. $\boldsymbol{B}$ shows the number of trials characterized by object rolls that were within $\pm 2^{\circ}$ from the vertical averaged across all subjects ( $\pm \mathrm{SE}$ ). ${ }^{* *} p<0.01$.

peak object roll, but it revealed a significant interaction between these two factors $\left(F_{(2,80)}=3.35, p<0.05\right)$. When subjects were given a visual cue about CM location, we found a main effect of $\mathrm{CM}$ location caused by longer latencies when the CM was on the left or right compared with the center $\left(F_{(2,94)}=9.73, p<0.01\right)$. No significant main effect of predictability condition or interaction between $\mathrm{CM}$ location and predictability condition was present. Post hoc analyses revealed no significant differences for any CM location for either cue experiment.

The no cue experiment ANOVA revealed a main effect of CM location $\left(F_{(2,106)}=14.75, p<0.001\right)$ and predictability condition $\left(F_{(1,53)}=66.72, p<0.001\right)$ and a significant interaction between these two factors $\left(F_{(2,106)}=5.85, p<0.01\right)$. Post hoc analysis showed that time to peak object roll was significantly longer in the random versus blocked condition when $\mathrm{CM}$ was located on the left (291 and $192 \mathrm{~ms}$, respectively; $p<0.001)$ and the right $(285$ and $185 \mathrm{~ms}$ and, respectively; $p<0.001$ ) but not when the CM was in the center (199 ms and169 ms, respectively; $p>0.05$ ).

\section{Across-trial adaptation of contact point modulation}

To capture the effect of explicit knowledge of object CM before the acquisition of implicit knowledge, we examined contact point modulation on the first object lift for each experimental condition (practice and first trial for blocked and random conditions, respectively). On the practice trial, the contact point of the index finger in the verbal cue was significantly higher when the CM was on the right compared with the left $\left(F_{(2,20)}=5.690, p<0.05\right)$. There were no significant differences found in the blocked condition of the visual cue experiment. For the first trial of the random condition, contact points were modulated between right and left CM locations in the verbal cue and visual cue experiments (verbal cue, $F_{(2,22)}=4.53,4.38$, and 4.97, for thumb, index, and middle, respectively; visual cue, $F_{(2,22)}=5.59$ for the thumb; all $p<0.05)$. This pattern of modulation resembles that described for data averaged across all trials for each CM location
(Fig. 2). These results suggest that modulation of contact points to $\mathrm{CM}$ in the random condition occurred from the very first object lift when a cue was provided, and thus explicit knowledge alone was sufficient to elicit this anticipatory control.

We found no significant differences in contact points between the first trials of any of the experimental sequences (all $p$ values $>0.40$ ). This result rules out possible effect(s) of learning transfer from one experiment/condition to the next.

\section{Across-trial and -experiment comparisons of object roll minimization}

As qualitatively described above (Fig. 4), in the blocked condition, peak object roll on the practice trial was significantly larger than that on the first experimental trial for each experiment (all $p$ values $<0.01$ ) (Fig. $8 A$ ). Therefore, subjects benefited from experiencing the external torque on the practice trial and were able to quickly adapt their force distribution to reduce object roll in the first experimental trial. However, peak object roll on the practice trial in the no cue experiment was not significantly different from that on the practice trial of either verbal cue or visual cue experiment (both $p>0.05$ ) (Fig. $8 B$ ). This indicates that availability of either a verbal or visual cue did not allow subjects to consistently minimize object roll significantly better than when no cue was available. Peak object roll in the practice trial in both cue experiments was significantly greater than that in the first experimental trial of the no cue experiment (Fig. 8C) (main effect of experiment, both $p<0.01$ ). Therefore, implicit knowledge of object CM enabled subjects to plan digit forces to object CM more accurately than when they were provided with only explicit knowledge of object CM.

In the random condition, subjects' ability to minimize object roll did not improve significantly as a function of repeated, nonconsecutive exposure to any of the CM locations for all experiments. No significant difference was found in peak object roll on the first versus second and first versus fifth trials for neither cue experiment $(p>0.05)$. Linear regression analyses performed on peak object roll versus trial for each CM revealed nonsignificant relations in $>97 \%$ of regressions. Therefore, subjects performed similarly from the first trial onward and thus were unable to retain and/or retrieve sensorimotor information gained through previous yet nonconsecutive trials (Fig. 4, white boxes).

Last, we performed linear regression analyses of peak object roll versus trial number to assess the existence of possible trends in object roll minimization that might have occurred as a function of practice (i.e., progressively smaller rolls with additional trials). This analysis was performed across all trials throughout the three experiments. Only 4 of 12 subjects exhibited significant negative trends in object roll performance, but the coefficients of determination $\left(r^{2}\right)$ of the linear fits were very weak (range of 0.02-0.08). Most importantly, three of these four subjects had started with the no cue experiment whose random condition was characterized by larger rolls than in the cue experiments (Figs. 4, 8 ). These analyses rule out significant transfer of implicit knowledge of object CM across conditions or experiments. 


\section{Discussion}

This study quantified the effect of visual and verbal cues about object CM location on anticipatory grasp control. Unlike previous studies, we allowed subjects to choose contact points because we reported recently that anticipatory grasp mechanisms are not limited to force modulation but include modulation of digit placement (Lukos et al., 2007). We hypothesized that subjects would be unable to use cues to plan digit placement and forces when CM was randomly changed. Surprisingly, this hypothesis was only partially supported by our findings, because cues enabled subjects to change the spatial distribution of contact points to object CM similarly in blocked and random conditions. However, subjects were able to minimize object roll to a greater extent for blocked conditions compared with random conditions even when cues were provided. To the best of our knowledge, this study is the first to provide evidence for separate mechanisms underlying sensorimotor memories of digit contact points and forces for grasping. This dissociation of the effects of cues on grasp kinematics versus kinetics is discussed in relation to neural mechanisms underlying the formation and use of sensorimotor memories. Because the effects of verbal and visual cues were similar, the discussion focuses on subjects' ability to use explicit knowledge regardless of the cue providing knowledge about CM location.

\section{Explicit knowledge does not allow accurate anticipatory force control} As hypothesized, in the random condition, explicit knowledge of CM location did not enable object roll minimization, hence accurate anticipatory control of digit forces, to the same extent as the blocked condition. This conclusion is supported by the significant main effect of predictability condition of average peak object roll (Fig. $5 B$ ), analyses of the number of trials characterized by very small rolls (Fig. $7 B$ ), and the adaptation of subjects' ability to minimize object roll (Fig. 8). For the latter analyses, we found (1) improvement in object roll minimization after acquisition of implicit knowledge regardless of the presence of cues (Fig. 8A), (2) similar peak object rolls during the practice trial of cue and no cue experiments (Fig. $8 \mathrm{~B}$ ), and (3) greater ability to minimize object roll after acquiring implicit knowledge of $\mathrm{CM}$ than on the very first manipulation with explicit knowledge (Fig. 8C).

These results are consistent with previous reports of subjects' inability to use arbitrary visual or verbal cues to anticipate digit forces as a function of object weight (Flanagan and Beltzner, 2000). Additionally, Turrell et al. (1999) reported a smaller gain in anticipatory force control as a function of impact force between a hand-held object and a pendulum when impact magnitude was verbally cued than when learned through practice. Similarly, Salimi et al. (2003) reported that subjects' were unable to (row $\boldsymbol{C}$ ).
$P_{\text {verbal }}$ vs. 1 ${ }^{\text {st }}$ verbal

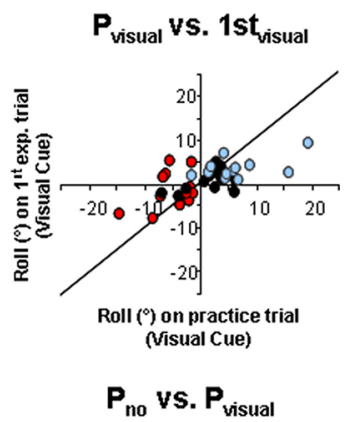

B $P_{\text {no }}$ vs. $P_{\text {verbal }}$
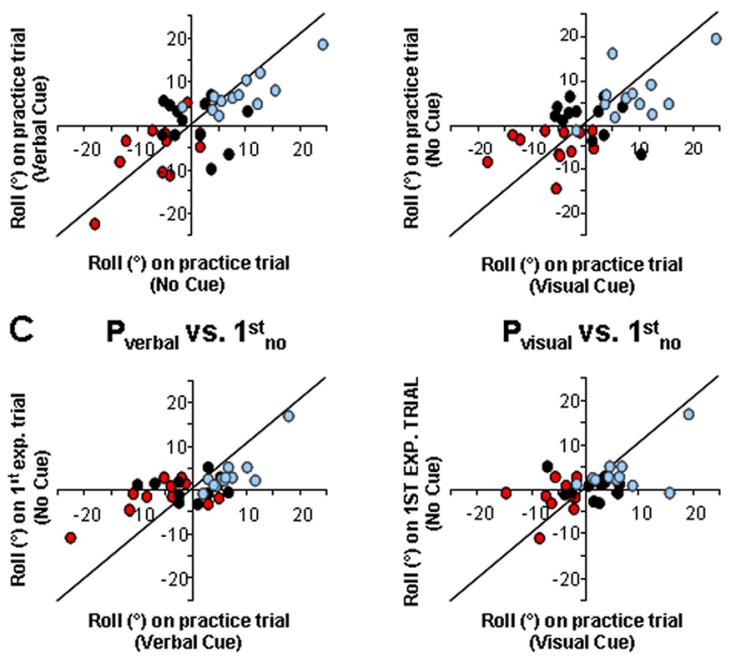

(') on practice

(Nisual Cue)

Figure 8. Object roll minimization on practice trial and first experimental trial. Row $A$ shows peak object roll during the blocked vs $1 \mathrm{st}_{\text {no, }}, \mathrm{P}_{\text {verbal }}$ vs $1 \mathrm{st}_{\text {verbal' }}$ and $\mathrm{P}_{\text {visual }}$ vs $1 \mathrm{st}_{\text {visual, }}$ respectively). Row $\boldsymbol{B}$ shows peak object roll during the blocked condition

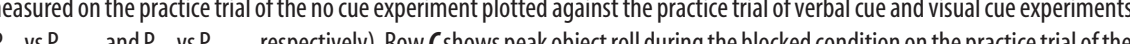

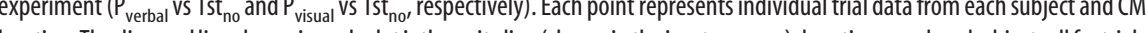
of peak object roll (i.e., ability to minimize object rolls) occurring from practice trial to first experimental trial (row A), on practice trial without versus with cues (row $\boldsymbol{B})$, and on the practice trial with cues versus first experimental trial of the no cue experiment

use visual cues about object CM for planning asymmetrical partitioning of digit forces. However, when subjects lifted a rotated L-shaped object for the first time after consecutive practice with the opposite CM location, forces were partitioned asymmetrically between the thumb and index finger. Yet, this effect of geometric cue was not as effective in eliciting accurate partitioning of digit forces as consecutive practice, this being indicated by significantly greater object rolls in the former condition (Salimi et al. 2003).

In contrast to the above studies, others have shown that subjects were able to use arbitrary visual cues about object weight to properly scale digit forces (Cole and Rotella, 2002; Nowak et al., 2007; Ameli et al., 2008; Chouinard et al., 2005). However, differences in the object property studied (i.e., CM vs weight) and the order of presentation of object properties might underlie some of the opposing results and thus prevent a direct comparison across studies. For example, in the study by Nowak et al. (2007), alternating two object weights on every trial could have allowed subjects to more easily predict the upcoming object weight, hence weakening the effect of object weight changes. Therefore, the cause(s) underlying the dis- 
crepancy in the results from these studies deserves additional investigation.

\section{Effective use of explicit knowledge for anticipatory grasp control}

Although cues were only partially effective in accurate anticipation of digit forces, they allowed subjects to modulate contact points as a function of object CM location, even when randomly changed from trial to trial, in a similar manner as in blocked CM presentations (Fig. 2). An "immediate" effect of cue was also found on the first lift, although this was primarily for cued random rather than blocked trials. We speculate that this finding was prompted by subjects' awareness of the experimental condition (random vs blocked) that could have caused them to rely on cues more heavily when they knew stable sensorimotor memories of object CM could not be built in the random condition.

The effectiveness of the cues on contact point modulation may have stemmed from subjects' knowledge of the upcoming object roll direction, i.e., associating object roll direction with the need to use an inverse relation of thumb and index finger (and less so for the other fingers) (Fig. 2) to better minimize object roll. Note that the same pattern was also elicited when knowledge of object CM could only be acquired implicitly, thus suggesting that this contact point distribution is optimal for controlling vertical orientation of the object during lift.

The above speculation that knowledge of torque direction is effective in allowing anticipatory control of contact points might lead to the expectation that digit force planning, too, could have been influenced to some degree. Indirect evidence for this is provided by two interrelated findings. First, the random conditions of the cue experiments were associated with statistically similar variances of peak object roll distribution for blocked and random conditions (Fig. 7A). Second, the results of time to peak object roll show that voluntary counteraction of the object roll occurred at longer latencies when its direction could not be anticipated (no cue random condition). In contrast, within each cue experiment, subjects initiated corrective responses for random conditions at similar latencies as those found for the blocked conditions, this being indicative of some degree of force planning even when $\mathrm{CM}$ was randomized.

\section{Sensorimotor memories: effect of implicit versus explicit knowledge of object properties}

We have shown recently that implicit knowledge gained from past manipulations enabled subjects to modulate contact points and minimize roll, whereas the inability to anticipate object $\mathrm{CM}$ led to no modulation of contact points and inappropriate force scaling (Lukos et al., 2007). This suggests that grasp kinematics and kinetics are planned in parallel. The present findings, however, point to a differential effect of cues on planning grasp kinematics versus kinetics. Specifically, although cues about randomly changed CM locations had an effect on (1) contact point distribution and (2) large object rolls and time to peak object roll, force planning was not as accurate as that resulting from implicit knowledge of object CM (Figs. 5B, 7B). Therefore, although parallel, it appears that the anticipatory mechanisms of grasp kinematics and kinetics can be processed independently. We speculate that anticipatory scaling of digit forces relies on implicit knowledge derived from consecutive manipulation to a greater extent than digit positioning does. Specifically, subjects were able to anticipate digit placement as a function of $\mathrm{CM}$ and were not influenced by CM randomization. This is a remarkable finding since studies of two-digit grasping have shown that anticipatory force scaling is biased by sensorimotor memories associated with the previous trial (Johansson and Westling, 1988; Gordon et al., 1993; Jenmalm and Johansson, 1997; Fellows et al., 1998, Witney et al., 2001).

\section{Neural mechanisms for anticipatory control of grasp kinematics and kinetics}

Virtual lesions induced by repetitive transcranial magnetic stimulation (rTMS) have been used to identify the cortical regions associated with anticipatory force control in response to implicit and explicit knowledge of object properties. Chouinard et al. (2005) found that rTMS of primary motor cortex selectively impairs subjects' ability to retrieve sensorimotor memories associated with previous manipulations but leaves the ability to use arbitrary visual cues unaffected. However, rTMS of dorsal premotor cortex had the opposite effect, thus stressing the specific role played by each region in processing implicit and explicit knowledge for grasp force planning.

Recent evidence suggests that contact points and forces are selectively affected by rTMS. Specifically, Davare et al. (2006) reported that rTMS of ventral premotor cortex interfered with consistent positioning of the thumb and index finger before object lift but did not affect the temporal coordination of grip forces. Opposite results, however, were found when rTMS was delivered to dorsal premotor cortex, indicating independent control of these two grasp components. Additional dissociations were found in the time domain: rTMS of anterior intraparietal area selectively affected hand shaping, but not force scaling, when delivered earlier during the reach and vice versa for stimulations delivered closer to the time of object contact ( $\mathrm{Da}-$ vare et al., 2007).

Although our behavioral data are consistent with evidence from rTMS studies of independent mechanisms for controlling grasp kinematics and kinetics, ongoing work is examining the formation and retrieval of their interrelated sensorimotor memories.

\section{References}

Ameli M, Dafotakis M, Fink GR, Nowak DA (2008) Predictive force programming in the grip-lift task: the role of memory links between arbitrary cues and object weight. Neuropsychologia 46:2383-2388.

Burstedt MK, Flanagan JR, Johansson RS (1999) Control of grasp stability in humans under different frictional conditions during multidigit manipulation. J Neurophysiol 82:2393-2405.

Chouinard PA, Leonard G, Paus T (2005) Role of the primary motor and dorsal premotor cortices in the anticipation of forces during object lifting. J Neurosci 25:2277-2284.

Cole KJ, Rotella DL (2002) Old age impairs the use of arbitrary visual cues for predictive control of fingertip forces during grasp. Exp Brain Res 143:35-41.

Davare M, Andres M, Cosnard G, Thonnard JL, Olivier E (2006) Dissociating the role of ventral and dorsal premotor cortex in precision grasping. J Neurosci 26:2260-2268.

Davare M, Andres M, Clerget E, Thonnard JL, Olivier E (2007) Temporal dissociation between hand shaping and grip force scaling in the anterior intraparietal area. J Neurosci 27:3974-3980.

Fellows SJ, Noth J, Schwarz M (1998) Precision grip and Parkinson's disease. Brain 121:1771-1784.

Flanagan JR, Beltzner MA (2000) Independence of perceptual and sensorimotor predictions in the size-weight illusion. Nat Neurosci 3:737-741.

Friedman J, Flash T (2007) Task-dependent selection of grasp kinematics and stiffness in human object manipulation. Cortex 43:444-460.

Gordon AM, Westling G, Cole KJ, Johansson RS (1993) Memory representations underlying motor commands used during manipulation of common and novel objects. J Neurophysiol 69:1789-1796. 
Jenmalm P, Johansson RS (1997) Visual and somatosensory information about object shape control manipulative fingertip forces. J Neurosci 17:4486-4499.

Johansson RS, Westling G (1988) Coordinated isometric muscle commands adequately and erroneously programmed for the weight during lifting task with precision grip. Exp Brain Res 71:59-71.

Johansson RS, Backlin JL, Burstedt MK (1999) Control of grasp stability during pronation and supination movements. Exp Brain Res 128:20-30.

Lukos J, Ansuini C, Santello M (2007) Choice of contact points during multidigit grasping: effect of predictability of object center of mass location. J Neurosci 27:3894-3903.

Nowak DA, Koupan C, Hermsdörfer J (2007) Formation and decay of sensorimotor and associative memory in object lifting. Eur J Appl Physiol 100:719-726.

Rearick MP, Santello M (2002) Force synergies for multifingered grasping: effect of predictability in object center of mass and handedness. Exp Brain Res 144:38-49.

Reilmann R, Gordon AM, Henningsen H (2001) Initiation and development of fingertip forces during whole-hand grasping. Exp Brain Res 140:443-452.

Salimi I, Hollender I, Frazier W, Gordon AM (2000) Specificity of internal representations underlying grasping. J Neurophysiol 84:2390-2397.

Salimi I, Frazier W, Reilmann R, Gordon AM (2003) Selective use of visual information signaling objects' center of mass for anticipatory control of manipulative fingertip forces. Exp Brain Res 150:9-18.

Turrell YN, Li FX, Wing AM (1999) Grip force dynamics in the approach to a collision. Exp Brain Res 128:86-91.

Witney AG, Vetter P, Wolpert DM (2001) The influence of previous experience on predictive motor control. Neuroreport 12:649-653. 